\title{
Case fatality and recovery rates of COVID-19 outbreak: Comparison between high, middle- and low-income countries
}

\author{
Ashis Talukder ${ }^{1}$ and Sheikh Mohammed Shariful Islam ${ }^{2,2}$ \\ ${ }^{1}$ Khulna University \\ ${ }^{2}$ Deakin University
}

July 2, 2020

\begin{abstract}
The number of cases and mortality from COVID-19 is changing rapidly worldwide. We analyzed the case fatality rate (CFR) and recovery rate (RR) from COVID-19 using recent data. By using the information of CFR and RR, we made a comparison between high and middle or low-income countries to understand the current global outbreak. We further ranked the countries based on their CFR and RR from higher to lower.
\end{abstract}

Title Page

Case fatality and recovery rates of COVID-19 outbreak: Comparison between high, middleand low-income countries

Running Head: COVID-19 case fatality and recovery rates

Ashis Talukder ${ }^{*}$, Sheikh Mohammed Shariful Islam²

${ }^{1}$ Statistics Discipline, Khulna University, Khulna-9208, Bangladesh.

${ }^{2}$ Institute for Physical Activity and Nutrition, Deakin University, Melbourne, VIC 3125, Australia.

${ }^{*}$ Corresponding Author: Ashis Talukder;E-mail address: ashistalukder3168@ku.ac.bd

Detailed Address of Corresponding Author:

Ashis Talukder

Assistant Professor

Statistics Discipline

Khulna University, Khulna-9208, Bangladesh

E-mail: ashistalukder3168@ku.ac.bd

Contact no.: +8801772063507

Case fatality and recovery rates of COVID-19 outbreak: Comparison between high, middleand low-income countries

The number of cases and mortality from COVID-19 is changing rapidly worldwide. We analyzed the case fatality rate (CFR) and recovery rate (RR) from COVID-19 using recent data. By using the information of CFR and RR, we made a comparison between high and middle or low-income countries to understand the current global outbreak. We further ranked the countries based on their CFR and RR from higher to lower. 
We considered 38 countries reporting at least 100 deaths on $12^{\text {th }}$ April 2020 by worldometer [1]. A Poisson distribution model (a probability distribution widely used to count data) was considered to estimate the overall case fatality and recovery rate from COVID-19 using the following formula:

$\mathrm{P}(x ; \mu)=\frac{e^{-\mu} \mu^{x}}{x !} ; x=0,1,2, \ldots \ldots$.

Here, $x$ represents the number of deaths or recovery due to COVID-19 and $\mu$ represents the fatality or recovery rate due to the disease. We used the following formula to calculate CFR and RR:

$$
\begin{gathered}
C F R=\frac{\text { Number of deaths due to COVID }-19}{\text { Total number of confirmed cases of COVID }-19} \\
R R=\frac{\text { Number of patients recovered from COVID }-19}{\text { Total number of confirmed cases of COVID }-19}
\end{gathered}
$$

The calculated rates and estimated value of the overall death and recovery rates with $95 \%$ confidence interval are shown in Supplementary Table 1 (see, supplementary material). Results show that CFR was relatively higher for Algeria (15.07\%) compared to other countries. The CFR for Italy, UK, Belgium, France, Netherland, and Spain was more than $10 \%$. Among these 38 countries, Russia $(0.86 \%)$ had the lowest CFR.

On the other hand, the recovery rate (RR) from COVID-19 was much higher in China (94.43\%) indicating that in China, people were recovering quickly from COVID-19 than other countries. Apart from China, only five countries (South Korea, Iran, Switzerland, Germany and Austria) showed RR more than 45\%. RR was relatively higher than CFR in most of the countries, which is encouraging (see, Figure 1). However, ten countries including Norway, Portugal, Ireland, Dominican Republic, Brazil, Philippines, Sweden, Indonesia, Netherland and the UK, the CFR was higher than RR (see, Figure 1). Among these ten countries, Ireland, the UK, Norway, Brazil and Netherland had the worst RR (less than one) compared to other countries. Moreover, the estimated death rate and recovery rate suggest that on an average, for every 100 confirmed COVID-19 patients, more than five patients might die [estimate $=5.86 ; 95 \%$ CI $(5.85,5.88)]$ and more than 20 patients will recover $[$ estimate $=20.22 ; 95 \%$ CI $(20.19,20.25)]$ from COVID-19.

Although the number of cases and deaths were higher in high-income countries, the case fatality rate was relatively high in most of the low-and middle-income countries (see, Figure 2). A possible reason for this difference may be the lack of national and health systems preparedness, lack of awareness about COVID-19 among the people and poor health facilities and infrastructures in these countries. Furthermore, it is a possibility that many cases and death remained undetected in these low-income countries due to lack of testing and diagnosis. Therefore, concerned authorities in low-and middle-income countries should consider performing more tests to correctly identify the COVID-19 patients and provide better symptomatic treatments to facilitate recovery since there exist no effective vaccines or medications for this disease [2]. Also, social isolation and lockdown policies may play a crucial role to reduce the current fatalities due to COVID-19 in low-and-middle-income countries like it did in some high-income countries.

\section{Funding Information}

No fund has been received

\section{Declaration of competing interest}

The author has no conflict of interest to disclosure

\section{References}

1. Worldometer. Available on: https://www.worldometers.info/coronavirus/

2. Wang $\mathrm{D}, \mathrm{Hu} \mathrm{B}, \mathrm{Hu} \mathrm{C}$, et al. Clinical characteristics of 138 hospitalized patients with 2019 novel coronavirus-infected pneumonia in Wuhan, China. JAMA. 2020; 323: 1061. 


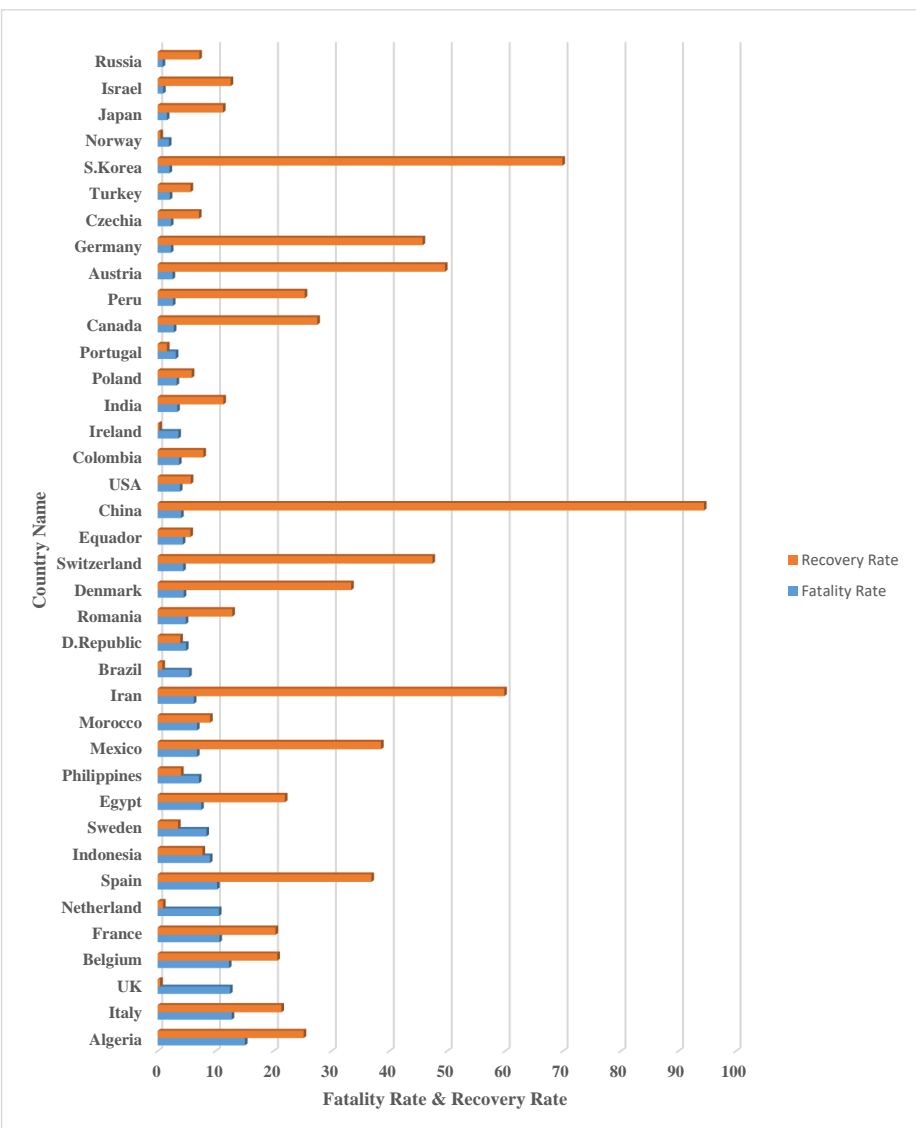

Figure 1: Graphical representation of Case Fatality Rate and Recovery Rate from COVID19 of different countries 